\title{
Findings from the 2021 Yearbook Section on Health Information Management
}

\author{
Meryl Bloomrosen', Eta S. Berner'2, Section Editors for the IMIA Yearbook Section on Health \\ Information Management \\ 1 Premier healthcare alliance, Washington, DC, USA \\ 2 Graduate Programs in Health Informatics, Department of Health Services Administration, \\ University of Alabama at Birmingham, Birmingham, AL, USA
}

\begin{abstract}
Summary
Objectives: To summarize the recent literature and research and present a selection of the best papers published in 2020 in the field of Health Information Management (HIM) and Health Informatics.

Methods: A systematic review of the literature for the IMIA Yearbook HIM section was performed by the two section editors with the help of a medical librarian. We searched bibliographic databases for HIM-related papers using both MeSH headings and keywords in titles and abstracts. A shortlist of the fifteen best candidate papers was first selected by section editors before being peer-reviewed by independent external reviewers.

Results: The three major themes of Health Information Exchange (transmitting, sharing, and accessing patient health-related data and information) (HIE), Data Quality, and Privacy and Security make up $80 \%$ of the fifteen papers, with individual papers on personal health records, information governance and the professionalism of the HIM field.

Conclusions: Traditional HIM concerns about HIM practice and workforce as well as issues about the data in electronic health records (EHRS) including data quality, coding, health information exchange among entities within the healthcare systems and privacy and confidentiality continue to be a large part of the HIM research literature. Although there was little research applying these themes to pandemic concerns, HIM professionals have the expertise to make ccontributions to public health informatics research and this research would benefit from their involvement.
\end{abstract}

\section{Keywords}

Health information management, health information exchange, data quality, privacy and security, electronic health records, public health informatics

Yearb Med Inform 2021:84-90

http://dx.doi.org/10.1055/s-0041-1726501

\section{Introduction}

The COVID-19 pandemic that dominated healthcare throughout most of 2020 highlighted many longstanding challenges and public health problems that have traditionally been key areas of focus for HIM professionals. These areas include the quality of the data in the medical records, including currency, completeness, accuracy of data and code assignments as well as the need for processes to securely exchange information among healthcare providers, across healthcare settings, and between public health agencies and healthcare providers. The traditional concerns of privacy and security became a prominent focus in the context of mobile applications (apps) to identify COVID-19 exposure, contact tracing, and so-called vaccination passports.

The HIM section survey paper by Massoudi and Sobolevskaia [1] explores these themes in the context of the pandemic. The survey paper also focuses on public health data, information systems, technology, and infrastructure, including workforce. In this review of the 2020 research related to Health Information Management (HIM), we explore these themes more broadly as exemplified by the group of papers selected as candidates to be the best papers of 2020 .

\section{Methods}

In January 2021, with the assistance of a medical librarian, the editors of the HIM section conducted a search of PubMed and Embase using both MeSH headings and keywords in the titles and abstracts with a focus on HIM, but with an additional focus of key areas of HIM interest in the context of COVID-19. The publication year was 2020. The search strategy was as follows. A search of PubMed was done first using the following search terms: ("Health Information Management" [mh] OR "Health Information Management" [tiab] OR "Health Information Management Journal" [ta] OR "J AHIMA" [ta]) AND 2020 [dp]. This search returned 156 articles. A search of Embase was then done using these terms: ("Health Information Management" [mh] OR "Health Information Management" [tiab] OR "Health Information Management Journal" [Journal] OR "J AHIMA" [ta]) AND 2020[dp]. This search returned 581 articles.

To add topics related to both COVID-19 and HIM, the following searches were done. In PubMed, the following search terms were used: (Privacy [mh] OR "Contact tracing"[mh] OR Privacy [ti] OR "Contact tracing" [ti] OR contact-detection [ti]) AND (2019-nCoV OR 2019nCoV OR COVID-19 OR SARS-CoV-2 OR ((wuhan AND coronavirus) AND 2019/12[dp]:2030[dp]) OR coronavirus OR "corona virus" OR coronavirinae OR coronaviridae OR betacoronavirus OR covid19 OR nCoV OR "CoV 2" OR CoV2 OR sarscov2 OR "novel CoV" OR "wuhan virus" OR ((wuhan OR hubei OR huanan) AND ("severe acute respiratory" OR pneumonia) AND (outbreak)) OR "Coronavirus" [mh] OR "Coronavirus Infections" [mh] OR "COVID-19" [Supplementary Concept] OR "severe acute respiratory syndrome coronavirus 2" [Supplementary Concept] OR "Betacoronavirus"[mh] OR "SARS2" OR "SARS-CoV-19" OR 
("severe acute respiratory" pneumonia outbreak) OR "novel cov" OR "sars cov2" OR ncov OR covid2019 OR ncov2019 OR ncov-2019 OR hcov19 OR hcov2019 OR hcov-19 OR hcov-2019 OR sars-cov-2 OR ((wuhan OR china OR Chinese OR novel) AND ("coronavirus" [mh] OR coronavirus OR coronaviruses OR corona-virus OR corona-viruses OR covid OR hcov)) OR ((2019[dp] OR 2020[dp]) AND (new[tiab] OR novel[tiab] OR pandemic[tiab] OR epidemic[tiab]) AND (coronavirus*[tiab] OR corona virus*[tiab]))) AND 2020[dp]. This search resulted in 728 articles. For Embase, the following search strategy was used: 'privacy'/exp OR 'contact examination'/exp OR 'contract tracing':ti OR 'contact detection':ti OR privacy:ti) AND ('covid 19'/exp OR 'coronavirus disease 2019'/exp OR 'covid 19 ':ti,ab OR ((covid NEAR/2 19):ti,ab) OR ((sars NEAR/2 'cov 2'):ti,ab) OR '2019 ncov':ti,ab OR ((wuhan:ti,ab OR hubei:ti,ab) AND coronavirus*:ti,ab) OR ([2019-2020]/ py AND (new:ti,ab OR novel:ti,ab OR pandemic:ti,ab OR epidemic:ti,ab) AND ('coronavirus infection'/exp OR coronavirus*:ti,ab OR 'corona virus*':ti,ab))) AND ('article'/ it OR 'article in press'/it OR 'review'/it) AND [2020-2020]/py." This search yielded 875 articles. After eliminating duplicates and articles lacking abstracts, there were 1,602 articles.

The 1,602 unique articles were rated by both section editors, who excluded articles that were opinion pieces, editorials, or reviews, or deemed not relevant to HIM. Each of the two section editors independently judged the relevance to the HIM field and the quality of the articles. Because the COVID-19 terms added many articles that were not relevant to HIM, to make the review task more manageable, articles that either co-editor rated as not appropriate were excluded automatically. The rest of the articles were discussed, and disagreements adjudicated to arrive at 15 articles that, based primarily on the abstracts, were judged to be of good quality. The full texts of these 15 articles were then rated independently by both section editors, two of the Yearbook editors, and two external peer reviewers.

The three 'Best Papers' were selected based on the consensus of reviewers that they should be included as one of the best papers. Other factors included having a high average rating from the reviewers, diversity of research approaches or focal areas and setting diversity. Below, we discuss the major themes of the fifteen research papers from 2020 that were candidates for being selected as a 'Best Paper for 2020'.

\section{Results}

The three major themes of Health Information Exchange (transmitting, sharing, and accessing of patient health-related data and information) (HIE), Data Quality, and Privacy and Security make up $80 \%$ of the fifteen papers, with individual papers on personal health records, information governance and the professionalism of the HIM field constituting the remaining $20 \%$. In a sense, data quality and privacy and security can be considered subthemes of HIE since they are a prerequisite for successful and trustworthy HIE. The three major themes will be discussed first, and the individual papers will be reviewed in the last section of this synopsis.

\subsection{Health Information Exchange}

Five of the best paper candidates, including one selected as Best Paper, addressed various aspects of HIE including technical capabilities, policies, and patient preferences related to data sharing. While most acute care and ambulatory settings in the US have had, since 2009, incentives for the adoption and use of

electronic health records (EHRs) and HIE [2], this has not been true for post-acute and long-term care settings, yet these settings represent a crucial partner for HIE. Thus, it is important to assess the current state of EHR adoption and HIE in these settings. Powell et al. used a mixed methods approach to examine technical capabilities and barriers to HIE in skilled nursing facilities [3]. This paper was designated one of the Best Papers and is described in more detail in the appendix, but briefly, the authors found that $95 \%$ of US nursing homes had EHRs and $46 \%$ were capable of some type of HIE. However, there were geographic differences in technological capabilities and there were also organizational, regulatory and user perception barriers that led to decreased HIE, even when the technical capabilities were adequate. These barriers will need to be addressed to achieve widespread and successful bi-directional HIE between long term and post-acute care settings and other providers.

User perceptions, technical capabilities and regulatory and organizational policies can interact with each other. For instance, in a study examining racial differences in preferences for opt-in or opt-out policies about data sharing and exchange, Turvey et $a l$. found racial differences in preferences among patients in the US Veterans Affairs hospitals, which at the time of the study had an opt-in policy [4]. Over half (56.8\%) of Caucasian respondents expressed preferences for an opt-out policy, while less than half $(38.3 \%-48.3 \%)$ of non-White respondents (Native Americans, African-Ameri-

Table 1 Best paper selection of articles for the IMIA Yearbook of Medical Informatics 2021 in the section 'Health Information Management'. The articles are listed in alphabetical order of the first author's surname.

Section

Health Information Management

- Cappetta K, Lago L, Potter J, Phillipson L. Under-coding of dementia and other conditions indicates scope for improved patient management: A longitudinal retrospective study of dementia patients in Australia. Health Inf Manag 2020:1833358319897928.

- Powell KR, Deroche CB, Alexander GL. Health data sharing in US nursing homes: a mixed methods study. J Am Med Dir Assoc 2021 May;22 (5):1052-59.

- Sheriffdeen A, Millar JL, Martin C, Evans M, Tikellis G, Evans SM. (Dis)concordance of comorbidity data and cancer status across administrative datasets, medical charts, and self-reports. BMC Health Serv Res 2020;20(1):858. 
cans, Latinos, and Asian/Pacific Islanders) preferred an opt-out policy. Apathy and Holmgren examined regulatory barriers and consent policies regarding data sharing and exchange of individual hospitals [5]. They found that in states with opt-in consent requirements for HIE, there was overall less HIE than in states that allowed opt-out consent for HIE. However, the researchers also found that these policies mainly affected the hospitals that were less technologically sophisticated. Among hospitals that were more sophisticated, state laws regarding optin or opt-out policies did not significantly create regulatory burdens, nor impact the volume of HIE.

In addition to hospital consent policies, there are differences in hospital choices on which, or how many, types of HIE and supporting mechanisms they can provide. Everson and Butler [6] examined the use of three types of HIE: secure messaging, provider portals and participation in an HIE organization. They found that from 2014 to 2016, there was an increasing use of all three methods, but there was also an increase in the use of multiple methods of HIE over that time period. They also found that the use of these methods individually increased the availability of outside information to providers and that the use of more methods increased availability even more. However, the study was unable to determine if the outside information, even though it was available, was accessed and used.

The studies described above were based on secondary analyses of large-scale datasets, in some cases followed up by qualitative interviews of key informants. Another approach to assessing patient perceptions related to information exchange was used by Esmaeilzadeh et al. [7]. The researchers provided scenarios depicting both structured and unstructured data for both physical and mental disorders to over 600 participants with self-reported physical or mental disorders via Amazon's Mechanical Turk system. Structured formats for information exchange were preferred for both types of disorders, although for different reasons. For physical disorders, structured formats were seen as providing higher quality information in terms of conciseness, understandability, and usefulness. For mental disorders, structured formats were perceived as more privacy-protecting. Both groups of patients were more likely to be willing to share their own information if it were in a structured format.

These studies examining different aspects of HIE illustrate that technological capabilities are a necessary, but insufficient, requirement for effective HIE. Attention to the HIE processes and approaches taken, user concerns and perceptions about those approaches and the overall regulatory environment affect the actual success of HIE, even with the appropriate technological infrastructure. Although these studies were not focused on the concerns specific to pandemic information sharing, they do provide useful information on the issues that need to be addressed in general and with public health and pandemic-specific information sharing. In addition to policies and perceptions, studies on data quality and privacy and security specifically provide insight that can be applied to pandemic concerns as well as post-pandemic data sharing.

\subsection{Data Quality}

The four papers among the fifteen best paper candidates that focused on data quality employed different methods and had different foci, but all found problems in data quality. A strength of each of these studies is that they were conducted across multiple hospitals, and one was conducted longitudinally as well. Thus, the problems in data quality are not unique to one hospital but appear to be more systemic. Mirhashemi et al. conducted an audit of ICD-10 coding across nine educational centers in Iran [8]. They found a 30\% error rate and advocated better training, more coordination between healthcare providers and coders, and an improved documentation process. A study by Cappetta et al., designated as a "Best Paper" and described in more detail in the appendix, examined the coding of a particular diagnosis, dementia [9]. The researchers studied the medical records over a five-year period of patients who had been diagnosed with dementia. Since dementia is a chronic disease that must be managed and is also expected to influence the treatment of other conditions, it would be expected that the diagnosis would continue to be assigned to patients over subsequent admissions. Instead, Cappetta et al. found that less than two-thirds of the patients had dementia included in diagnoses over the subsequent 12 months and that the documentation of this diagnosis decreased over time, so that at the end of five years, it was coded only about $54 \%$ of the time.

While the studies of Mirhashemi et al. and Cappetta et al. focused specifically on coding of the data in EHRs, two other studies examined the concordance of different data sources. Sheriffdeen et al., in another of the Best Papers, examined the agreement of comorbidity data for prostate cancer patients among medical records, administrative data and patient self-report [10]. Nshimyiryo et al. studied the agreement between individual facility reports and data in a national Health Management and Information System (HMIS) in Rwanda [11]. Both studies found discrepancies between data sources, but some types of data were more discrepant than others. Sheriffdeen et al., similar to Cappetta et al., found that co-morbidities were particularly problematic. Accurate co-morbidity data are important in general, but especially for risk prediction during a pandemic. These data, across different diseases, and in different countries, underscore the challenges in obtaining accurate data in the process of HIE.

\subsection{Privacy and Security}

The three papers that addressed privacy and security each addressed different aspects. Aldughayfiq and Sampalli reviewed published articles and websites to evaluate the architectures and privacy and security protections of e-prescribing systems from eight different countries [12]. They found a great deal of variability, especially whether systems were centralized or decentralized and whether they had capabilities for new methodologies or technologies such as blockchain or artificial intelligence that could enhance privacy.

Zhou and Parmanto used a mixed methods design to study what users of mobile health apps preferred in terms of privacy protections when using mHealth apps that 
contained sensitive health information [13]. Even though the users may have decided themselves to use the apps, they wanted methods to protect their privacy, not just of the information contained in the apps, but even the fact that they were using the app. When offered a choice of five methods to protect privacy, they preferred including apps that were not unique to a particular disease and they wanted the ability to hide the app. In particular, users wanted privacy protection methods that they could customize themselves.

While the two previously described studies examined privacy protections in two specific types of systems (e-prescribing and mHealth), Walden et al. used a survey with different breach scenarios to study how privacy officers in hospitals respond to breaches [14]. What was interesting about the results is that breach reporting decisions appeared to be done on a case-by-case basis and that in some scenarios, over half of respondents said they would not report them. Contrary to what might be expected, the researchers found that the privacy officers with more education or more credentials were less likely to report the more ambiguous situations where it was not clear whether the breach was required to be reported or how severe the breach was.

These papers highlight several considerations regarding privacy and security of health information. With the range of new and innovative technologies, tools and approaches regarding data collection, transmission, and storage, along with increased digitalization of health information, there is a heighted need to address ongoing and increasingly complex technical, technological, public policy, legal, and ethical issues. Additionally, the COVID-19 pandemic heightened the awareness of the need to share data globally and raised significant questions about data timeliness, accuracy, and transparency - what could be shared, with whom, when and under what conditions. HIM professionals and health informaticians, along with other stakeholders, including researchers, clinicians, policymakers, and public health officials and practitioners, face challenges and opportunities to ensure data availability and accessibility, while balancing requirements for privacy and security.

\subsection{Other Topics of Interest to the HIM Profession}

In addition to expertise and interest in the areas of HIE, data quality, and privacy and security, HIM professionals have a long-standing interest in Personal Health Records (PHRs), information governance and the HIM profession itself. The last three articles of the set of fifteen candidate best papers address these topics. Alsahafi et al. investigated receptivity to using PHRs in Saudi Arabia [15]. Saudi Arabia is in the process of developing a national PHR system and the study was designed to assess readiness for it. The researchers utilized the Unified Theory of Acceptance and Use of Technology (UTAUT) as their theoretical framework. They made some modifications and adjustments to some of the elements in the theory by adding measures of eHealth literacy and deleting the constructs related to actual use, since the PHR was not yet developed. They found that the model explained $56 \%$ of the variance in intention to use the PHR. Performance expectancy, effort expectancy, social influence and eHealth literacy were key determinants of intention to use a PHR. These data are useful for several reasons. They will assist Saudi Arabia (and potentially other countries) in understanding key factors affecting the use of the system they are developing. In addition, the use of a theoretical model can help others do similar assessments of readiness for use that will allow comparability across settings.

As the number of health informatics applications in clinical settings has been increasing, HIM professionals have been instrumental in bringing the topic of information governance to the attention of the health informatics and clinical community. Kwan et $a l$. focused their study on an examination of the current state of health information governance in hospitals in Victoria, Australia [16]. They also compared their results to a similar study done in the US. Their response rate was $42 \%$. One of the key findings was that only half of their sample of hospitals had a formal information governance program, which was like the 2014 US rate of $43 \%$. However, $80 \%$ of respondent hospitals expressed interest in starting such a program, which again was similar to the $90 \%$ response rate in the US.
Although there is some concern that less than half of the hospitals responded to the survey, it is encouraging to see interest in formal information governance growing in multiple countries.

The last article by Thye et al. described the development of an instrument to measure the professionalism of HIM professionals [17]. The researchers found that HIM professional activities comprised strategic, tactical, and operational information management. The instrument was validated in three different countries.

What is significant about all three of these studies is that they address concerns that are important to HIM professionals worldwide. The studies either used a theoretical model that has been used in multiple settings, replicated studies from other countries, or validated their instruments across multiple countries. These data provide some assurance of the generalizability of their results as well as instruments that can be used internationally.

\section{Conclusion}

All the themes and topics of individual articles that have been discussed have implications for HIM and governance that are exacerbated during emergencies such as natural disasters and pandemics. Interoperability between health care and public health information and data systems is critical. Seamless HIE between laboratories and healthcare providers, between providers and immunization registries, and between providers and public health agencies is key for public health protection, prevention, tracking and mitigation. The need for robust data is key to safe, effective, and efficient patient care and treatment, and may be compromised if co-morbidities are not well-documented. Accurate, timely, and trustworthy information is also essential and impacts the ability to deploy interventions and control disease spread during a pandemic. Attitudes of consumers to privacy and security as well as consent issues are key for contact tracing and use of technologies, such as mobile apps for exposure notification. One of the major reasons the COVID-19 terms 
were added to the search strategy for this section was to discover research linking more traditional HIM concerns (such as data quality and coding; patient matching and identification; information governance; and privacy and security of data) with response to the pandemic. However, as can be seen from the selection of the candidate best papers, none of them focused explicitly on the COVID-19 context. Only one of them mentioned that their study had implications for COVID-19. The field of HIM has great potential to contribute to the broader public health field as these papers and the section survey paper illustrate. We believe that HIM professionals should be engaged in issues that go beyond traditional HIM boundaries, so that the information management knowledge, expertise, and perspective can be brought to bear more broadly on society's emerging and ongoing health and health related challenges. Public health practices and operations would benefit from the application of HIM and informatics principles, research, analysis, innovation, and discovery.

\section{References}

1. Massoudi BL, Sobolevskaiya D. Keep moving forward: health informatics and information management beyond the COVID-19 pandemic. Yearb
Med Inform 2021:75-83.

2. Centers for Medicare \& Medicaid Services. Promoting Interoperability Programs [Internet]. 2021. Available from: https://www.cms.gov/ Regulations-and-Guidance/Legislation/EHRIncentivePrograms

3. Powell KR, Deroche CB, Alexander GL. Health Data Sharing in US Nursing Homes: A Mixed Methods Study. J Am Med Dir Assoc 2021;22(5):1052-9.

4. Turvey CL, Klein DM, Nazi KM, Haidary ST, Bouhaddou O, Hsing N, et al. Racial differences in patient consent policy preferences for electronic health information exchange. J Am Med Inform Assoc 2020;27(5):717-25.

5. Apathy NC, Holmgren AJ. Opt-in consent policies: potential barriers to hospital health information exchange. Am J Manag Care 2020;26(1):e14-e20.

6. Everson J, Butler E. Hospital adoption of multiple health information exchange approaches and information accessibility. J Am Med Inform Assoc 2020;27(4):577-83.

7. Esmaeilzadeh P, Mirzaei T, Dharanikota S. The impact of data entry structures on perceptions of individuals with chronic mental disorders and physical diseases towards health information sharing. Int J Med Inform 2020;141:104157.

8. Mirhashemi SH, Ramezanghorbani N, Asadi F, Rangraz MH. Auditing the accuracy of medical diagnostic coding based on international classification of diseases, tenth revision. Iranian Red Crescent Med J 2020;22(9).

9. Cappetta K, Lago L, Potter J, Phillipson L. Under-coding of dementia and other conditions indicates scope for improved patient management: A longitudinal retrospective study of dementia patients in Australia. Health Inf Manag 2020:1833358319897928.

10. Sheriffdeen A, Millar JL, Martin C, Evans M,
Tikellis G, Evans SM. (Dis)concordance of comorbidity data and cancer status across administrative datasets, medical charts, and self-reports. BMC Health Serv Res 2020;20(1):858.

11. Nshimyiryo A, Kirk CM, Sauer SM, Ntawuyirusha E, Muhire A, Sayinzoga F, et al. Health management information system (HMIS) data verification: A case study in four districts in Rwanda. PLoS One 2020;15(7):e 0235823.

12. Aldughayfiq B, Sampalli S. Digital Health in Physicians' and Pharmacists' Office: A Comparative Study of e-Prescription Systems' Architecture and Digital Security in Eight Countries. OMICS 2021;25(2):102-22.

13. Zhou L, Parmanto B. User Preferences for Privacy Protection Methods in Mobile Health Apps: A Mixed-Methods Study. Int J Telerehabil 2020;12(2):13-26.

14. Walden A, Cortelyou-Ward K, Gabriel MH, Noblin A. To report or not to report health care data breaches. Am J Manag Care 2020;26(12):e395-e402.

15. Alsahafi YA, Gay V, Khwaji AA. Factors affecting the acceptance of integrated electronic personal health records in Saudi Arabia: The impact of e-health literacy. Health Inf Manag 2020:1833358320964899.

16. Kwan H, Riley M, Prasad N, Robinson K. An investigation of the status and maturity of hospitals' health information governance in Victoria, Australia. Health Inf Manag 2020:1833358320938309.

17. Thye J, Esdar M, Liebe JD, Jahn F, Winter A, Hübner U. Professionalism of Information Management in Health Care: Development and Validation of the Construct and Its Measurement. Methods Inf Med 2020;59(S 01):e1-e12.

\section{Correspondence to:}

Meryl Bloomrosen

E-mail:meryl_bloomrosen@premierinc.com 
Appendix: Content Summaries of Best Papers for the Health Information Management Section of the 2021
IMIA Yearbook

Powell KR, Deroche CB, Alexander GL

Health data sharing in US nursing homes: a mixed methods study

J Am Med Dir Assoc 2021 May;22 (5): 1052-59

Efforts to achieve nationwide interoperability in the US have been ongoing for several years in part due to federal legislation and regulation. Some providers, such as long term and post-acute care providers did not receive incentive payments for implementing electronic health records. The authors studied nursing homes' capability for data sharing and nursing home leaders' perceptions of data sharing with other health care facilities and with residents and family members. The authors explore longstanding challenges to improve data access by patients and their caregivers as well as provider-to-provider data sharing and exchange across sites of care. This was an exploratory mixed methods study. The authors performed a secondary analysis of data from a national survey of 815 nursing home administrators in the United States and used a survey developed to measure nursing home information technology (IT) adoption. The authors used descriptive statistics and logistic regression models to examine the relationship between health data sharing capabilities and nursing home characteristics such as location, bed size, and type of ownership. Additionally, between November 2018 and December 2019 , researchers conducted qualitative interviews with nursing home administrators. Interviews included questions about processes for sharing data with residents and family members and perceptions of data sharing with other clinical partners (e.g., hospitals and other entities). Perceived barriers to data sharing included privacy and security concerns, transparency and control, fear of lawsuits, and organizational factors which slowed the uptake of technology.
These organizational factors included an overregulated and punitive environment, perceptions about lack of interest among residents and providers, available and accessible resources, and other constraints (including financial, workflow, and technical issues), such as lack of patient portal availability through the health IT vendor. Perceived benefits of data sharing included improved communication and care planning and being able to anticipate future demand. The authors found that nursing homes varied greatly in their technological capabilities and perceptions about what and with whom they could share information. The COVID-19 pandemic highlighted the need for data collection and reporting about quality-of-care in nursing homes, testing and immunization of patients and staff. The pandemic also demonstrated the need for health information technology and electronic health records to help address infection prevention, and mitigation. Powell and colleagues include recommendations for additional research and public policies to address health IT gaps and challenges. Addressing these gaps and shortcomings will improve the secure exchange of information during public health emergencies, as well as on a more routine basis.

\section{Cappetta K, Lago L, Potter J, Phillipson L}

Under-coding of dementia and other conditions indicates scope for improved patient management: A longitudinal retrospective study of dementia patients in Australia

\section{Health Inf Manag 2020:1833358319897928}

The importance of the quality, accuracy, and consistency of clinical coding of medical information in hospitals is important for various use cases including payment, resource allocation, surveillance, epidemiological research, prevention, treatment, and to inform policy. This paper by Cappetta and colleagues was a longitudinal study of coding quality of dementia after the initial diagnosis to examine the implications for patient management and quality of care when dementia was not coded ("under coding") given a prior confirmed diagnosis of dementia. The researchers sought to inform future intervention studies to improve identification and management of dementia in hospitals. The population-based retrospective cohort study was conducted in a regional local health district of New South Wales, Australia with five hospitals. This study described rates of dementia coding over the 5 years (the study period was from 1 July 2006 to 30 June 2015 with a 5-year lookback period from 1 July 2001) after an initially coded admission for dementia. The study also identified unintended consequences related to lack of clinical coding (such as the potential under-management of dementia) and identified patient subgroups at risk of having inaccurate or incompletely coded diagnoses. The diagnoses were recorded using the International Classification of Diseases, Australian modification (ICD10-AM Ninth Edition). The researchers found that dementia was coded in $63.9 \%$ of admissions in the 12 months following the index admission for dementia and that the coding of dementia decreased to $53.7 \%$ after 5 years. They also reported that patients were $20 \%$ more likely to have dementia actively managed when it co-occurred with delirium. The paper highlights the relationship of data accuracy and clinical documentation completeness. Coding accuracy relies on robust clinical documentation and the absence of documentation (and related coding) raises questions about gaps in care delivery, patient management, quality of care and patient safety. The authors offer recommendations to address under-coding of chronic conditions and improve identification and management of dementia through dementia-specific care, enhanced clinical protocols, and other interventions.

\section{Sheriffdeen A, Millar JL, Martin C, Evans M, Tikellis G, Evans SM}

(Dis)concordance of comorbidity data and cancer status across administrative datasets, medical charts, and self-reports

\section{BMC Health Serv Res 2020;20(1):858}

As Sherffdeen and colleagues note, risk-adjustment for co-morbidities often requires data from sources that were originally designed for other purposes; therefore it is important to understand the underlying foundational principles and definitions of 
the datasets and the reliability of different data sources. In Australia, prostate cancer represents the second leading cause of cancer-related mortality in males. The Prostate Cancer Outcome Registry-Victoria (PCORVic) was developed in 2009 as a clinical quality registry, to measure and report on quality of care, using benchmarking of performance at a clinician and hospital level. The researchers used a retrospective cohort study design to study the completeness and accuracy of co-morbidity documentation as reflected in different data sources. The authors studied the level of concordance for same-patient comorbidity data extracted from administrative data sets (coded from ICD-10 AM), from the medical record, and data self-reported by men with prostate cancer who had undergone a radical prostatectomy between January 2017 and April 2018 at one of six convenient hospitals. The authors analyzed diseases based on the conditions included within the Charlson Comorbidity Index and compared comorbidities across the three data sources. Concordance was calculated using percentage agreement and the kappa statistic. The paper reports on the level of statistical concordance between the medical chart and administrative datasets; the medical chart and patient self-report data; and administrative data and patient self-report data. The researchers also included a summary of the concordance of comorbidity data across the three data sources. The study notes that agreement between comorbidity data collected by the Victorian Admitted Episodic Dataset, medical charts, and self-reports by men who have undergone a radical prostatectomy varied across the analyzed comorbidities. The authors identified discrepancies between (coded) administrative data sets and the medical charts and noted the need to further explore the impacts of coding guidelines and practices. They also found discrepancies between patient self-reported data and the other datasets which might highlight a need for better patient education or improved communication between patients and providers. Comorbidity data are important for accurate monitoring of risks and understanding the accuracy of data sources is critical to data use. The findings about the data quality of various sources are highly relevant to the HIM domain and functions. HIM is concerned with the reliability of coded data and the completeness and accuracy of the documentation to support coding. There are unintended consequences of incomplete and inaccurate data sources including documentation and coding. Recognizing that data completeness, accuracy, definitions, and formats may vary by sources is also relevant to the COVID-19 pandemic since multiple data sources (such as medical records, laboratory test results, case reports, immunization registries, patients, and caregivers) across sites of care are critical for public health surveillance, care delivery, disease management and analytics. Recognizing and reconciling discrepancies in data reporting, data and interoperability standards, and definitions is also essential to information sharing and exchange between health care and public health use cases. 